\title{
Citrus flavanones upregulate thyrotroph Sirt1 and differently affect thyroid Nrf2 expressions in old-aged Wistar rats
}

Marko Miler $^{1 *}$, Jasmina Živanović ${ }^{1}$, Vladimir Ajdžanović ${ }^{1}$, Dragan Milenkovic ${ }^{2,3}$, Ivana Jarić $^{1,4}$, Branka Šošić-Jurjević ${ }^{1}$, Verica Milošević ${ }^{1}$

${ }^{1}$ Department of Cytology, Institute for Biological Research "Siniša Stanković"- National Institute of Republic of Serbia, University of Belgrade, Belgrade, Serbia

${ }^{2}$ Université Clermont Auvergne, INRAE, UNH, F-63000 Clermont-Ferrand, France

${ }^{3}$ Division of Cardiovascular Medicine, University of California Davis, 95616 Davis,

California, USA

${ }^{4}$ Animal Welfare Division, Vetsuisse, University of Bern, Bern, Switzerland

*Correspondence to: Marko Miler, PhD

Institute for Biological Research "Siniša Stanković"- National Institute of Republic of Serbia, University of Belgrade

142 Despot Stefan Blvd.

11060 Belgrade, Serbia

Phone: +381112078 321

Fax: +381 112761433

E-mail: marko.miler@ibiss.bg.ac.rs 
<smiles>COc1ccc(C2CC(=O)c3c(O)cc(O)cc3O2)cc1O</smiles>

Figure S1 Structure of naringenin and hesperetin.
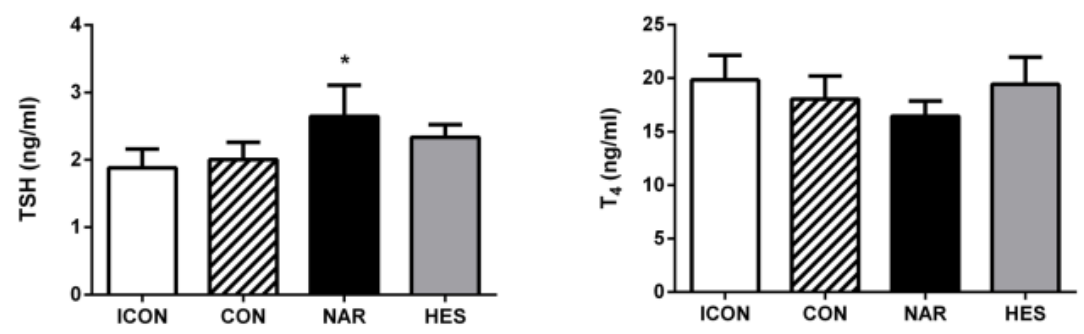

Figure S2 Serum level of thyroid-stimulating hormone (TSH) and thyroxin (T4) in intact control (ICON), control sunflower oil-treated (CON), naringenin- (NAR) and hesperetin(HES) treated rats. All of the values presented mean $\pm \mathrm{SD}(n=6)$; statistics: one way ANOVA, Dunett's multiple comparison post hoc test, $* \mathrm{p}<0.05$ versus CON rats. Reprinted from: Acta Histochemica, 119(3), Marko Miler, Ivana Jarić, Jasmina Živanović, Vladimir Ajdžanović, Nasta Tanić, Verica Milošević, Branka Šošić-Jurjević, Citrus flavanones mildly interfere with pituitary-thyroid axis in old-aged male rats, 292-301, Copyright (Mar 08, 2019), with permission from Elsevier, License Number 4544171222583. 

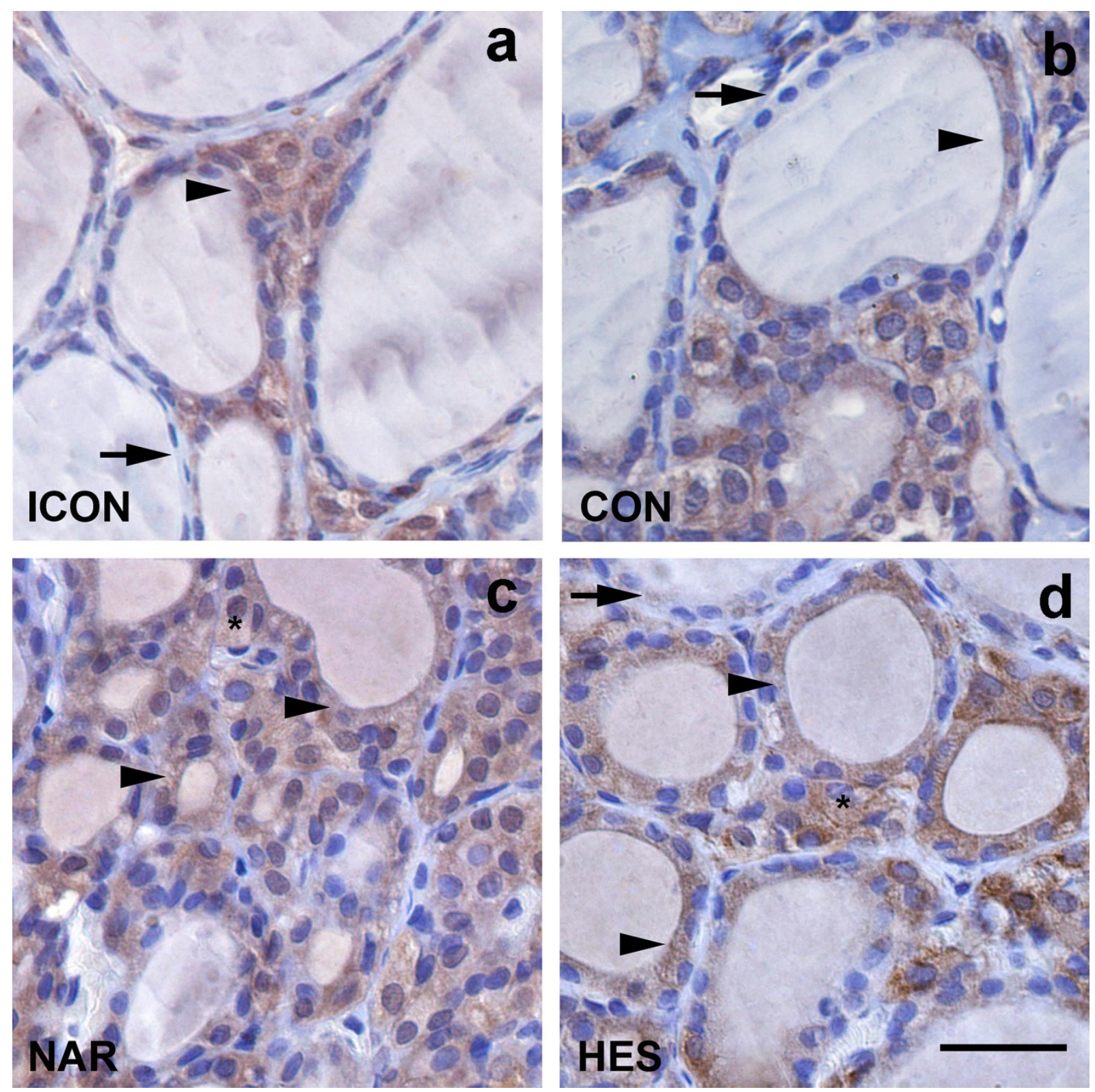

Figure S3 Effects of naringenin (NAR) and hesperetin (HES) on vascular endothelial growth factor (VEGF) in thyroids of old-aged rats. VEGF distribution and localization in: a) intact control (ICON), b) control sunflower oil- (CON), c) NAR- and d) HES- treated old-aged rats. Black arrows show absence of VEGF localization, while arrow heads show presence of VEGF signal in thyrocytes. $40 \times$ magnification, bar $=25 \mu \mathrm{m}$. 
a

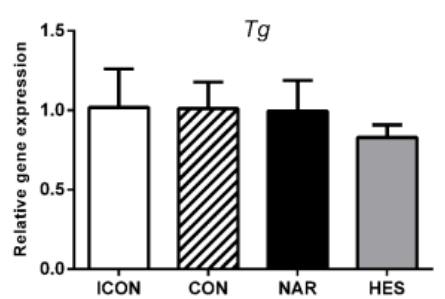

b

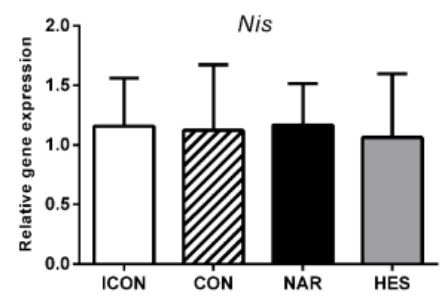

Figure S4 Gene expression of a) thyroglobulin (Tg) and b) sodium-iodide symporter (NIS) in thyroids of: intact control (ICON), control sunflower oil- (CON), NAR- and HES- treated oldaged rats. The each value represents mean $\pm \mathrm{SD}, \mathrm{n}=6$; statistics: one way ANOVA, Dunett's multiple comparison post hoc test, $* \mathrm{p}<0.05$ versus $\mathrm{CON}$ rats. 
Table S1 List of antibodies used in immunoflorescent (IF), immunohistochemical (IHC) and Western blot (WB) analysis.

\begin{tabular}{|c|c|c|c|c|c|}
\hline \multicolumn{6}{|c|}{ Primary antibodies used } \\
\hline & Name & Cat. num. & Origin & Dilution & Manufacturer \\
\hline \multirow{2}{*}{ IF } & TSH & AFP-1274789 & Rabbit & $1: 1000$ & Bethesda, MD, USA \\
\hline & Sirt1 & GTX82793 & Mouse & $1: 800$ & GeneTex, CA, USA \\
\hline \multirow{2}{*}{ IHC } & TPO & sc-376876 & Mouse & $1: 400 / 1: 500^{*}$ & Santa Cruz, Italy \\
\hline & VEGF & $a b 46154$ & Rabbit & $1: 100$ & \multirow{7}{*}{ Abcam, UK } \\
\hline \multirow{7}{*}{ WB } & SOD1 & ab13498 & \multirow{6}{*}{ Rabbit } & \multirow{5}{*}{$1: 2000$} & \\
\hline & SOD2 & ab13533 & & & \\
\hline & CAT & ab16731 & & & \\
\hline & GPx & ab22604 & & & \\
\hline & GR & ab16801 & & & \\
\hline & Nrf2 & ab92946 & & $1: 1000$ & \\
\hline & GAPDH & G2267 & Mouse & $1: 10000$ & Sigma-Aldrich, USA \\
\hline \multicolumn{6}{|c|}{ Secondary antibodies used } \\
\hline \multirow{2}{*}{ IF } & Alexa Fluor 488 & A-21206 & Donkey & $1: 500$ & $\begin{array}{c}\text { Thermofisher, CA, } \\
\text { USA }\end{array}$ \\
\hline & Alexa Fluor 647 & A-31571 & Donkey & $1: 400$ & $\begin{array}{c}\text { Thermofisher, CA, } \\
\text { USA }\end{array}$ \\
\hline \multirow{2}{*}{ IHC } & $\begin{array}{l}\text { Anti-mouse, } \\
\text { HRP labeled }\end{array}$ & Ab6820 & Donkey & \multirow{2}{*}{$1: 100$} & Abcam, UK \\
\hline & $\begin{array}{l}\text { Anti-rabbit, } \\
\text { HRP labeled }\end{array}$ & P0399 & Swine & & Dako, Denmark \\
\hline \multirow{2}{*}{ WB } & $\begin{array}{l}\text { Anti-mouse, } \\
\text { HRP labeled }\end{array}$ & $7076 \mathrm{~S}$ & \multirow{2}{*}{ Donkey } & \multirow{2}{*}{ 1:20000 } & \multirow{2}{*}{$\begin{array}{c}\text { Cell Signaling, MA, } \\
\text { USA }\end{array}$} \\
\hline & $\begin{array}{l}\text { Anti-rabbit, } \\
\text { HRP labeled }\end{array}$ & $7074 \mathrm{~S}$ & & & \\
\hline
\end{tabular}

*Dilution used in Western blot analysis. 
Table S2 Sequence of primer list used in quantitative PCR analysis

\begin{tabular}{|c|c|}
\hline Gene & Primer sequence \\
\hline$T s h$ & $\begin{array}{l}\text { f: 5'-TACAGAGACTTCACCTACAGA-3' } \\
\text { r: 5'-GGCAACGGGGTAGGAGAAATA-3' }\end{array}$ \\
\hline Sirt1 & $\begin{array}{l}\text { f: 5'-TGTTTCCTGTGGGATACCTGA-3' } \\
\text { r: 5'-TGAAGAATGGTCTTGGGTCTTT-3' }\end{array}$ \\
\hline Tpo & $\begin{array}{l}\text { f: 5'-TTGGATCTGGCATCACTGAACTT-3' } \\
\text { r: 5'-ATCTTGTTGACCATGCTTCTGTTG-3' }\end{array}$ \\
\hline$T g$ & $\begin{array}{l}\text { f: 5'-CCGGATATTGCAGAGACGAT-3' } \\
\text { r: 5'-GGCAGCTTGGGATATATGGA-3' }\end{array}$ \\
\hline Nis & $\begin{array}{l}\text { f: 5'-GGTGTCATCAGTGGGCCTCTA-3' } \\
\text { r: 5'-CCCGTGTCCATTCCAGAACTG-3' }\end{array}$ \\
\hline Sodl & $\begin{array}{l}\text { f: 5'-AAGCGGTGAACCAGTTGTG-3' } \\
\text { r: 5'-CCAGGTCTCCAACATGCC-3' }\end{array}$ \\
\hline Sod2 & $\begin{array}{l}\text { f: 5'-GGTGGAGAACCCAAAGGAGA-3' } \\
\text { r: 5'-AGCAGTGGAATAAGGCCTGT-3' }\end{array}$ \\
\hline Cat & $\begin{array}{l}\text { f: 5'-CCAGCGACCAGATGAAGCA-3' } \\
\text { r: 5'-TGGTCAGGACATCGGGTTTC-3' }\end{array}$ \\
\hline Gpx & $\begin{array}{l}\text { f: 5'-TATAGAAGCCCTGCTGTCCA-3' } \\
\text { r: 5'-CAAGCCCAGATACCAGGAA-3' }\end{array}$ \\
\hline Gpx2 & $\begin{array}{l}\text { f: 5'-ATATTGTCCCCTTGCCTTCC-3' } \\
\text { r: 5'-ACCTACCCCAGACTTAGAGC-3' }\end{array}$ \\
\hline$G r$ & $\begin{array}{l}\text { f: 5'-ACGAGGAAGACGAAATGCGTGATG-3' } \\
\text { r: 5'-AGGATGAATGGCGA CGCTATTGTC-3' }\end{array}$ \\
\hline Nrf2 & $\begin{array}{l}\text { f: 5'-GAGCGGGAGAAATCACACAGAATG-3' } \\
\text { r: 5'-CAGGAGCTGCATGCACTCATCG-3' }\end{array}$ \\
\hline Nqol & $\begin{array}{l}\text { f: 5'-ATTGTATTGGCCCACGCAGA-3' } \\
\text { r: 5'-GATTCGACCACCTCCCATCC-3' }\end{array}$ \\
\hline Gapdh & $\begin{array}{l}\text { f: 5'-GTGGACCTCATGGCCTACAT-3' } \\
\text { r: 5'-GGATGGAATTGTGAGGGAGA-3' }\end{array}$ \\
\hline
\end{tabular}

